\title{
First observations and magnitude measurement of Starlink's Darksat
}

\author{
J. Tregloan-Reed ${ }^{1}$, A. Otarola ${ }^{2}$, E. Ortiz ${ }^{3}$, V. Molina ${ }^{1}$, J. Anais $^{1}$, R. González ${ }^{1}$, J. P. Colque ${ }^{1}$, and E. Unda-Sanzana ${ }^{1}$ \\ ${ }^{1}$ Centro de Astronomía (CITEVA), Universidad de Antofagasta, Avenida U. de Antofagasta, 02800 Antofagasta, Chile \\ e-mail: jeremy.tregloanreed@uantof.cl \\ 2 TMT International Observatory, 100 West Walnut Street, Pasadena, CA 91124 USA \\ 3 Departamento de Física, Universidad de Antofagasta, Avenida Angamos 601, Antofagasta, Chile
}

Received 13 March 2020 / Accepted 15 April 2020

\begin{abstract}
Aims. We measured the Sloan $g^{\prime}$ magnitudes of the Starlink's STARLINK-1130 (Darksat) and 1113 low Earth orbit (LEO) communication satellites to determine the effectiveness of the Darksat darkening treatment at $475.4 \mathrm{~nm}$.

Methods. Two observations of the Starlink's Darksat LEO communication satellite were conducted on 2020/02/08 and 2020/03/06 using Sloan $r^{\prime}$ and $g^{\prime}$ filters, respectively. A second satellite, STARLINK-1113, was observed on 2020/03/06 using a Sloan $g^{\prime}$ filter. The initial observation on 2020/02/08 was a test observation conducted when Darksat was still in the process of manoeuvring to its nominal orbit and orientation. Based on the successful test observation, the first main observation took place on 2020/03/06, along with an observation of the second Starlink satellite.

Results. The calibration, image processing, and analysis of the Darksat Sloan $g^{\prime}$ image gives an estimated Sloan $g^{\prime}$ magnitude of $7.46 \pm 0.04$ at a range of $976.50 \mathrm{~km}$. For STARLINK-1113, an estimated Sloan $g^{\prime}$ magnitude of $6.59 \pm 0.05$ at a range of $941.62 \mathrm{~km}$ was found. When scaled to a range of $550 \mathrm{~km}$ and corrected for the solar and observer phase angles, a reduction by a factor of two is seen in the reflected solar flux between Darksat and STARLINK-1113.

Conclusions. The data and results presented in this work demonstrate that the special darkening coating used by Starlink for Darksat has darkened the Sloan g' magnitude by $0.77 \pm 0.05$ mag when the range is equal to a nominal orbital height $(550 \mathrm{~km})$. This result will serve members of the astronomical community who are actively modelling the satellite mega-constellations to ascertain their actual impact on both amateur and professional astronomical observations. Both concurrent and subsequent observations are planned to cover the full optical and NIR spectrum using an ensemble of instruments, telescopes, and observatories.
\end{abstract}

Key words. techniques: photometric - light pollution - methods: observational

\section{Introduction}

In May 2019, Starlink, a subsidiary of SpaceX, launched their first batch of 60 low Earth orbit (LEO) communication satellites. Due to the extremely bright apparent magnitude of these satellites, caused by their very low orbits following launch and their clustering in "trains", this launch and subsequent launches have caused major concern among both the amateur and professional astronomical communities (see IAU press release, 2019/06/03 and IAU press release, 2020/02/12). Hainaut \& Williams (2020) recently examined the impact on ESO telescopes in the optical and NIR and suggest that the greatest impact from the LEO communication satellites will be on ultra-wide imaging exposures from large telescopes (e.g. National Science Foundation's Vera C. Rubin Observatory, formerly known as LSST). A second study, carried out by McDowell (2020), concludes that LEO satellites will have a significant impact on twilight astronomy and observations that use wide fields of view with long exposures.

Starlink, via an application submitted by SpaceX, has received approval by the US FCC (Federal Communications Commission) to have 12000 Starlink LEO communication satellites in orbit (FCC Authorization Report and FCC statement). SpaceX have since applied to the international radio-frequency regulator for a further 30000 Starlink LEO communication satellites to be placed in low orbit ${ }^{1}(328 \mathrm{~km}$ to $580 \mathrm{~km})$. The threat to ground-based optical and radio astronomical research from the Starlink mega-constellations is still being evaluated by the AAS and IAU. Starlink is working with with the astronomical community to reduce the brightness of the Starlink satellites ${ }^{2}$.

Following these discussions, Starlink undertook an attempt to make the satellites dark enough so that they do not saturate the Charles Simonyi telescope's camera detectors at the Vera C. Rubin Observatory. At 02:19 UTC on January 7 2020, Starlink launched its third batch of LEO communication satellites and one of them, STARLINK-1130 (nicknamed "Darksat") was given an experimental darkening treatment on one side to reduce its reflective brightness (SpaceX press kit, January 2020), although the exact details of this treatment have not been published.

In this Letter, we present the first ground-based observations of the Darksat (international designation: 44932) LEO communication satellite (observed on 2020/02/08 and 2020/03/06) ${ }^{3}$, along with an additional non-darkened Starlink LEO communication

\footnotetext{
1 Spacenews.com accessed on 2020/03/01.

2 The Atlantic accessed on 2020/02/07.

3 The FITS files are available from the author by request.
} 
Table 1. Log of observations presented for STARLINK-1130 (Darksat) and STARLINK-1113.

\begin{tabular}{lccc}
\hline \hline & 1130 & 1130 & 1113 \\
& Darksat & Darksat & \\
\hline Date (J2000) & $2020 / 02 / 08$ & $2020 / 03 / 06$ & $2020 / 03 / 06$ \\
Time (UTC) & $00: 52: 30$ & $00: 30: 22$ & $00: 15: 26$ \\
Filter & Sloan $r^{\prime}$ & Sloan $g^{\prime}$ & Sloan $g^{\prime}$ \\
Exposure time (s) & 8.0 & 1.5 & 2.0 \\
Altitude $(\mathrm{km})$ & 471.62 & 563.48 & 563.97 \\
Range $(\mathrm{km})$ & 516.73 & 976.50 & 941.62 \\
Azimuth $\left(^{\circ}\right)$ & 314.37 & 236.12 & 215.69 \\
Elevation $\left({ }^{\circ}\right)$ & 64.95 & 31.48 & 33.19 \\
Airmass & 1.10 & 1.90 & 1.81 \\
RA (Sat) & 041222.71 & 021336.07 & 020938.66 \\
Dec (Sat) & -054221.46 & -402455.62 & -573901.94 \\
RA (Sun) & 212434.99 & 230806.44 & 230804.13 \\
Dec (Sun) & -151249.45 & -053330.31 & -053344.79 \\
Sun-Zenith angle $\left({ }^{\circ}\right)$ & 109.2 & 110.0 & 106.7 \\
Sun-Satellite angle $\left(^{\circ}\right)$ & 83.7 & 54.7 & 64.3 \\
\hline
\end{tabular}

satellite, STARLINK-1113 (international designation: 44926) (observed on 2020/03/06), as a comparison.

\section{Observations}

Two observations of Darksat (STARLINK-1130) were conducted on 2020/02/08 and 2020/03/06, whereas STARLINK1113 was observed on 2020/03/06, using the Chakana $0.6 \mathrm{~m}$ telescope at Universidad de Antofagasta's Ckoirama observatory in northern Chile $(24.1 \mathrm{~S}, 69.9 \mathrm{~W})$. The instrument was a FLI ProLine 16801 camera, operated with a Sloan $r^{\prime}(2020 / 02 / 08)$ and $g^{\prime}(2020 / 03 / 06)$ filter. In this setup, the CCD covers a field of view of $32.4 \times 32.4$ arcmin with a pixel scale of $0.47 \operatorname{arcsec}_{\text {pixel }}{ }^{-1}$.

Our telemetry code first retrieves the Starlink two-line element (TLE) data from the Celestrak ${ }^{4}$ website. Then, using the coordinates of the observatory, it calculates the ephemerides of the satellite and of the Sun. Table 1 gives an observing log of the observations presented in this work, including the test observation.

\section{1. $2020 / 02 / 08$ test observation}

An initial test observation was performed on 2020/02/08, to determine the accuracy of our telemetry and positional code for Starlink satellites and the effectiveness of our observing technique.

With these ephemerides, we were able to preset the telescope pointing to the required coordinates prior to the arrival of the satellite. To give a high precision measurement of the Darksat trail in the acquired test image, no binning of the detector was used. The readout time for a full-frame, non-binned image of the FLI ProLine 16801 camera is $\approx 11.2 \mathrm{~s}$. We calculated that Darksat had an sky-projected velocity of $\approx 1350 \operatorname{arcsecs~s}^{-1}$. For a $11.2 \mathrm{~s}$ readout time, Darksat would end up traversing over 15000 arcsec (over 32000 pixels). Therefore, to reduce the likelihood of Darksat passing through the field of view during readout, the exposure time was set at $8 \mathrm{~s}$, minimising the dead-time

\footnotetext{
4 https://celestrak.com/NORAD/elements/supplemental/ starlink.txt
}

ratio. After the successful test observation, it was decided for future observations to reduce the exposure time in an attempt to capture the full satellite trail within the field of view.

\section{2. $2020 / 03 / 06$ main observations}

The observation of Darksat conducted on 2020/03/06 was the first observation, during which Darksat had reached both its nominal orbital height $(550 \mathrm{~km})$ and orientation. Therefore, it is not straightforward to directly compare the results from the two observations since once the Starlink satellite has reached its nominal orbit, its orientation changes and it becomes significantly less visible from the ground (SpaceX press kit, January 2020).

Once a Starlink satellite had completed its manoeuvre to its nominal orbit, the TLE data showed that at the apex of the Starlink satellite's trajectory, the sky-projected velocity of the satellite was on the order of a few degrees per second. In an attempt to obtain a full satellite track wholly within the field of view, the observing strategy used in the test observation would need to be changed when using a field of view of $32.4 \times$ 32.4 arcmin combined with a readout time of $11.2 \mathrm{~s}$. To help obtain a full satellite track within the field of view, three adjustments to the observing strategy were undertaken. First, to reduce the sky-projected velocity of the satellite, a lower elevation was selected for the observations. The second change was to reduce the readout time (down to $1.7 \mathrm{~s}$ ) by using $4 \times 4$ binning of the detector. Thirdly, the exposure times (see Table 1) were selected based on the sky-projected velocity of the satellites $(1.5 \mathrm{~s}$ for Darksat and $2 \mathrm{~s}$ for STARLINK-1113) to allow the full trail to be shorter than the field of view. These changes were partially successful with the Darksat observation. However, knowing the predicted ephemerides, even if the track is not fully enclosed in the detector, makes it possible for the satellite's magnitude to be estimated. To give the best approximation to the visual band $(550 \mathrm{~nm})$, the observations of both Darksat and STARLINK1113 conducted on 2020/03/06 used a Sloan $g^{\prime}$ filter $(475.4 \mathrm{~nm}$, FWHM $138.7 \mathrm{~nm}$ ).

\subsection{Data reduction}

The raw Flexible Image Transport System (FITS) files were calibrated by removing the instrumental signature from the images by subtracting the bias and dividing by the flat-field. The flux of the comparison stars was measured using standard aperture photometry.

In examining a section of the Darksat satellite trail from the test observation, it becomes clear that there is a sharp cutoff between the trail point spread function (PSF) and sky background along the trail length (see Fig. 1). From this we set two parallel boundary lines along the trail length and calculated the total integrated flux after subtracting the sky background.

The integrated flux of the satellite trail was then converted into magnitude using the calibration obtained from the comparison stars.

\section{Data analysis}

The test observation (2020/02/08) contained three suitable comparison stars. However, a review of the literature revealed that the magnitudes had only been determined in the Johnson passbands (Tycho-2 catalogue: Høg et al. 2000; Second US Naval Observatory CCD Astrograph Catalog: Zacharias et al. 2004). To convert the Johnson magnitudes to the Sloan $r^{\prime}$ passband, we used the 


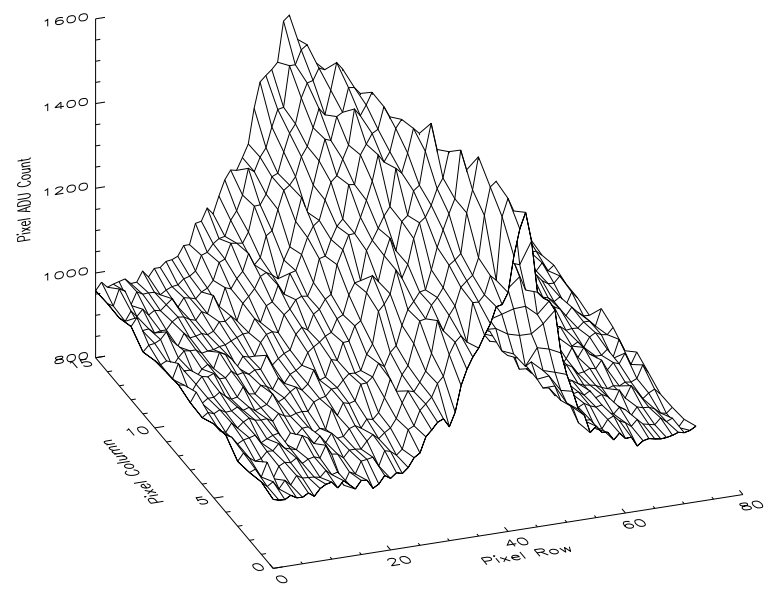

Fig. 1. Section of the Darksat trail from the test observation, obtained on 2020/02/08. The section shows a peak pixel count of $\approx 1600$ and a satellite trail PSF width of 24 pixels. The section is from $x(3678: 3750), y(1815: 1830)$ of the main FITS.

Table 2. Comparison stars used in this work.

\begin{tabular}{lccc}
\hline \hline $\begin{array}{l}\text { Star } \\
\text { Name }\end{array}$ & $\begin{array}{c}\text { Comparison } \\
\text { Star }\end{array}$ & $\begin{array}{c}\text { Sloan } r^{\prime} \\
\text { Mag. }\end{array}$ & $\begin{array}{c}\text { Sloan } g^{\prime} \\
\text { Mag. }\end{array}$ \\
\hline \multicolumn{4}{c}{ STARLINK-1130: 2020/02/08 } \\
\hline BD-05 848 & 1 & $9.18 \pm 0.07$ & - \\
HD 26528 & 2 & $9.27 \pm 0.04$ & - \\
$\mathrm{V}^{*}$ BZ Eri & 3 & $9.67 \pm 0.06$ & - \\
\hline \multicolumn{5}{c}{ STARLINK-1130: 2020/03/06 } \\
\hline CD-40 570 & - & $10.92 \pm 0.01$ \\
CD-40 571 & - & $10.74 \pm 0.04$ \\
\hline \multicolumn{5}{c}{ STARLINK-1113: 2020/03/06 } \\
\hline TYC 8489-723-1 & 1 & - & $11.62 \pm 0.01$ \\
TYC 8489-699-1 & 2 & - & $11.90 \pm 0.01$ \\
\hline \multicolumn{5}{c}{}
\end{tabular}

colour equation: $r^{\prime}=V-0.42(B-V)+0.11$ (Table 1: Jester et al. 2005). The comparison stars in the Darksat and STARLINK1113 observations conducted on 2020/03/06 have reported Sloan $g^{\prime}$ magnitudes (Munari et al. 2014), which allowed a direct calculation of the Darksat and STARLINK-1113 apparent magnitudes in the Sloan $g^{\prime}$ passband. The details of the comparison stars used in this work are given in Table 2.

Figure 2 shows the FITS images of the observations presented in this work, with the comparison stars listed in Table 2 labelled accordingly. Since the Starlink satellite trails in the observations were not wholly within the field of view, only an upper and estimated magnitude could be determined from the observations. The upper magnitude limit was calculated using the measured trail lengths. This leads to a fainter limit of the magnitude for the Starlink satellites. The estimated magnitude was determined by comparing the measured and predicted ephemerides trail lengths, giving an ephemeridesestimated magnitude. The results are presented in Table 3.

For the Darksat observation conducted on 2020/03/06, the measured and predicted ephemerides trail lengths are in agreement within their 1- $\sigma$ uncertainties, indicating that we successfully observed the full trail length in the image. However, when inspecting the trail in the image, the trail ends a pixel width from the CCD edge, making it difficult to confirm this. Therefore, we provide both the estimated and fainter limit magnitude of Darksat.
For the test observation, we measured the trail length to be $3412 \pm 58$ pixels, while the ephemerides predicted 22900 pixels for an eight second exposure. This leads to a correction by a factor of $\approx 6.7$, or $2 \mathrm{mag}$, as reported in Table 3 . For a comparison to the observations conducted on 2020/03/06 using a Sloan $g^{\prime}$ filter, we converted the test magnitude from Sloan $r^{\prime}$ to $g^{\prime}$. We used the colour equation, $g^{\prime}-r^{\prime}=1.05(B-V)-0.23$ from Fukugita et al. (1996), with a solar, $B-V=0.65$ (Allen 1973). This gives an estimated Sloan $g^{\prime}$ magnitude of $\approx 2.8$ for the test observation.

The calculated magnitudes given in Table 3 for the respective observations each have a different range $(r)$ to the Starlink satellite observed. The orbital height $\left(H_{\mathrm{orb}}\right)$ becomes the range when the satellite passes at the local zenith to the observer. Therefore, discerning the reflectivity ratio between Darksat and other Starlink satellites requires that the magnitude is normalised to the nominal orbital height, $550 \mathrm{~km}$ scaled using $+5 \log (r / 550)$. In addition, the magnitude needs to be corrected for the solar and observer phase angles, $\theta$ and $\phi$, respectively. The observer phase angle is the angle between the observer and the unit normal of the Earth facing surface of the satellite and is approximated by:

$\phi=\arcsin \left(\frac{\eta}{H_{\mathrm{orb}}} \sin \alpha\right)$,

where $\eta$ is the straight line distance between the observer and the satellite footprint (nadir) and $\alpha$ is the elevation. The solar and observer phase angles of Darksat and STARLINK-1113 are given in Table 4.

While the effects from the two phase angles on a complex body like a Starlink satellite is difficult to model precisely, most of the light observed is diffused. Therefore, we can approximate the effect by using a bidirectional reflectance distribution function (BRDF). Without empirical observations of the BRDF for the Starlink satellites, we can only provide an estimated value by using a parametrised BRDF model from Minnaert (1941). Consequently, we estimate the ratio $(R)$ of the solar phase attenuation between Darksat and STARLINK-1113 with:

$R=\left(\frac{\cos \theta_{1130} \cos \phi_{1130}}{\cos \theta_{1113} \cos \phi_{1113}}\right)^{k-1}$,

where $k$ is the Minnaert exponent and ranges from 0 to 1 and $k=1$ represents a perfect Lambertian surface.

Using $k=0$ gives $R \approx 0.8$ while, if $k=1$ then $R=1$, for the $\theta$ and $\phi$ of the observations on 2020/03/06. When $k=0.5$ (e.g. a dark surface Stamnes et al. 1999), then $R \approx 0.9$, which is in agreement to a first order approximation of the solar phase attenuation for a diffusing sphere, $(1+\cos \theta) / 2$ (Hainaut \& Williams 2020). If we assume $k=0.5$ then the solar and observer phase angles would make Darksat appear 0.11 mag darker than STARLINK-1113, prior to any darkening treatment.

In Table 4 we report the estimated magnitudes observed on 2020/03/06 after correction for the solar and observer phase angles, then normalised to a range of $550 \mathrm{~km}$. We do not include the test observation from 2020/02/08. As mentioned in Sect. 2, after the manoeuvring phase, the orientation of Darksat changes. Hence, the two observations of Darksat are of two different surfaces and this would require too many assumptions to give an accurate result.

The final results given in Table 4 show that at a range of $550 \mathrm{~km}$ and after correction for the solar and observer phase angles, Darksat is dimmer than STARLINK-1113 by $0.77 \pm$ 0.05 mag in the Sloan $g^{\prime}$ passband. This indicates that Darksat is twice as dim as STARLINK-1113. 

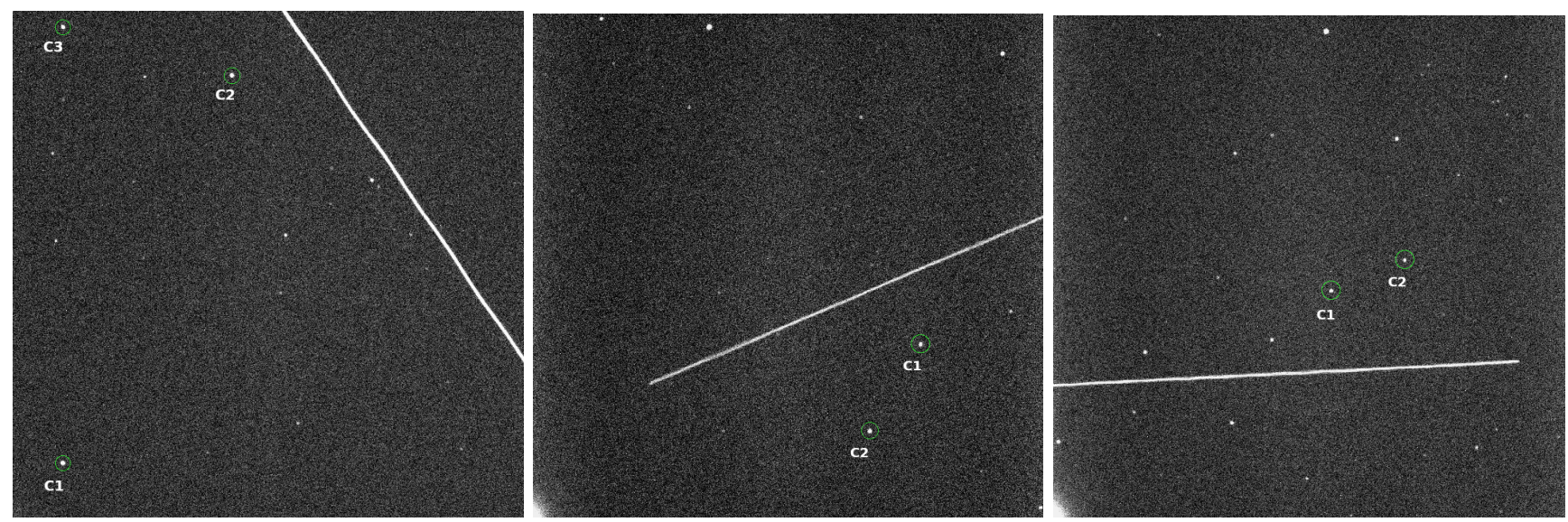

Fig. 2. FITS images of STARLINK-1113 and the two Darksat trails presented in this work. The comparison stars are numbered according to Table 2. Left: Darksat: 2020/02/08. Middle: Darksat: 2020/03/06. Right: STARLINK-1113: 2020/03/06.

Table 3. Results of the observations presented in this work for Darksat and STARLINK-1113.

\begin{tabular}{|c|c|c|c|c|}
\hline Star & $\begin{array}{c}\text { Lower } \\
\text { diff mag }\end{array}$ & $\begin{array}{c}\text { Estimated } \\
\text { diff mag }\end{array}$ & $\begin{array}{l}\text { Fainter } \\
r^{\prime} \text { mag }\end{array}$ & $\begin{array}{c}\text { Estimated } \\
r^{\prime} \text { mag }\end{array}$ \\
\hline \multicolumn{5}{|c|}{ STARLINK-1130: 2020/02/08 } \\
\hline BD-05 848 & $-4.88 \pm 0.08$ & $-6.88 \pm 0.09$ & $4.30 \pm 0.11$ & $2.30 \pm 0.11$ \\
\hline HD 26528 & $-4.82 \pm 0.05$ & $-6.82 \pm 0.07$ & $4.45 \pm 0.06$ & $2.45 \pm 0.08$ \\
\hline \multirow[t]{2}{*}{$\mathrm{V}^{*}$ BZ Eri } & $-5.36 \pm 0.07$ & $-7.36 \pm 0.09$ & $4.31 \pm 0.09$ & $2.31 \pm 0.11$ \\
\hline & & & $4.39 \pm 0.05$ & $2.38 \pm 0.06$ \\
\hline Star & $\begin{array}{c}\text { Lower } \\
\text { diff mag }\end{array}$ & $\begin{array}{c}\text { Estimated } \\
\text { diff mag }\end{array}$ & $\begin{array}{l}\text { Fainter } \\
g^{\prime} \text { mag }\end{array}$ & $\begin{array}{c}\text { Estimated } \\
g^{\prime} \text { mag }\end{array}$ \\
\hline \multicolumn{5}{|c|}{ STARLINK-1130: 2020/03/06 } \\
\hline CD-40 570 & $-3.46 \pm 0.04$ & $-3.47 \pm 0.04$ & $7.46 \pm 0.05$ & $7.45 \pm 0.05$ \\
\hline \multirow{2}{*}{ CD-40 571} & $-3.27 \pm 0.06$ & $-3.28 \pm 0.06$ & $7.48 \pm 0.10$ & $7.47 \pm 0.10$ \\
\hline & & & $7.47 \pm 0.04$ & $7.46 \pm 0.04$ \\
\hline \multicolumn{5}{|c|}{ STARLINK-1113: 2020/03/06 } \\
\hline TYC 8489-723-1 & $-4.73 \pm 0.04$ & $-4.91 \pm 0.06$ & $6.89 \pm 0.04$ & $6.60 \pm 0.06$ \\
\hline \multirow[t]{2}{*}{ TYC 8489-699-1 } & $-5.08 \pm 0.06$ & $-5.26 \pm 0.08$ & $6.86 \pm 0.06$ & $6.58 \pm 0.08$ \\
\hline & & & $6.88 \pm 0.04$ & $6.59 \pm 0.05$ \\
\hline
\end{tabular}

Notes. The results in bold are the weighted means from the individual results from each comparison star.

Table 4. Estimated magnitude of Darksat and STARLINK-1113 after correction for the solar and observer phase angles, then normalised to a range of $550 \mathrm{~km}$ (one airmass), including the sky-projected angular velocity along with the observed and estimated trail lengths.

\begin{tabular}{lcccccc}
\hline \hline $\begin{array}{l}\text { Starlink } \\
\text { satellite }\end{array}$ & $\begin{array}{c}\text { Observed } \\
\text { range }(\mathrm{km})\end{array}$ & $\begin{array}{c}\text { Solar phase } \\
\text { angle }\left(^{\circ}\right)\end{array}$ & $\begin{array}{c}\text { Observer phase } \\
\text { angle }\left(^{\circ}\right)\end{array}$ & $\begin{array}{c}\text { Angular speed } \\
\left.(\operatorname{arcsec~s})^{-1}\right)\end{array}$ & $\begin{array}{c}\text { Est. trail length } \\
(\operatorname{arcsec})\end{array}$ & $\begin{array}{c}\text { Estimated } \\
\text { scaled mag. }\end{array}$ \\
\hline 1130 (Darksat) & 976.50 & 54.7 & 50.6 & $1075 \pm 29$ & $1615 \pm 40$ & $6.10 \pm 0.04$ \\
1113 & 941.62 & 64.3 & 47.2 & $1033 \pm 27$ & $2066 \pm 45$ & $5.33 \pm 0.05$ \\
\hline
\end{tabular}

\section{Summary and discussion}

The successful test observation of the Starlink's Darksat confirm that the ephemerides computed from the publicly available TLE are accurate enough in position and timing to acquire satellite tracks with a professional telescope.

The first observation conducted on 2020/02/08 confirmed that the Starlink satellites are extremely bright $(\approx 2.4 \mathrm{mag}$, see Table 3) during the deployment phase (at $474 \mathrm{~km}$ orbital height). The magnitude was well within the naked-eye sensitivity of a casual observer. During the deployment phase, the Starlink satellites were in groups of 60 and form a train. It is expected that up to three or four satellite trains will be seen in any single night ${ }^{5}$ and will, therefore, have an impact on the natural darkness of the sky (see International Dark sky Association Press Release; Gallozzi et al. 2020).

The results from the observation of Darksat conducted on 2020/03/06 gives an estimated magnitude in the Sloan $g^{\prime}$ passband of $7.46 \pm 0.04$ at a range of $976.50 \mathrm{~km}$. The observation of STARLINK-1113 conducted on 2020/03/06 gives an estimated

5 nasapaceflight. com accessed on 2020/04/11. 
magnitude in the Sloan $g^{\prime}$ passband of $6.59 \pm 0.05$ at a range of $941.62 \mathrm{~km}$. This measurement at an elevation of $33.19^{\circ}$ validates the photometric model used by Hainaut \& Williams (2020), which predicts magnitude 6.6 to 6.7 in these conditions. When scaled to a range of $550 \mathrm{~km}$ and to an elevation of $90.0^{\circ}$, our result shows that the brightness of STARLINK-1113 is closer to their fainter estimations. To ascertain a ratio between the reflective flux between Darksat and STARLINK-1113, the magnitude must be corrected for the solar and observer phase angles and for the range normalised to the orbital height or one airmass. This shows that Darksat is $\approx 2$ times dimmer than STARLINK-1113. This value should be treated with caution, however. We report an estimated value from using a parametrised BRDF model from Minnaert (1941) and setting the Minnaert exponent to that of a dark surface (i.e. $k=0.5$ ). To obtain an accurate BRDF measurement will require multiple observations of Darksat and other Starlink satellites during a single trajectory path. Only then can different empirical reflectance BRDF models (e.g. Phong BRDF: Phong 1975; Lewis BRDF: Lewis 1994) be compared to the empirical measurements of Darksat and other Starlink satellites.

The results from this work show that Darksat is invisible to the naked eye, even under optimal conditions. However, this reduction does not meet the requirement needed to mitigate the effects that low orbital mega-constellation LEO communication satellites will have on ultra-wide imaging exposures from large telescopes, such as the National Science Foundation's Vera C. Rubin Observatory (formerly known as LSST). To help mitigate the impact from electronic ghosts in ultra-wide imaging exposures would require a satellite to be 15 times dimmer than a standard Starlink LEO communication satellite, which would approximately reach down to the 8th magnitude (see LSST Statement). However, Darksat is the first response on the part of Starlink with regard to the impacts of mega-constellations of LEO communication satellites on both amateur and professional astronomy. From what we understand, Starlink is studying other methods to decrease the brightness of its satellites, which may hopefully be deployed in an upcoming launch.

The observations presented in this work, taken when Darksat had reached its nominal orbit (2020/03/06) and orientation, are from a single passband (Sloan $g^{\prime}$ ). At the time of writing, further observations had just been completed at the Chakana $0.6 \mathrm{~m}$ telescope at Universidad de Antofagasta's Ckoirama observatory, in
Sloan $r^{\prime}$ and $i^{\prime}$; whilst on the same nights, observations of Darksat and STARLINK-1113 were conducted in the NIR ( $J$ and $K s$ bands) using VIRCAM (VISTA InfraRed CAMera) on the $4.0 \mathrm{~m}$ VISTA telescope, ESO Paranal, Chile. It is envisaged that the new data will provide a measurement of the change in reflectivity of Darksat as a function of wavelength, from the optical to NIR. Subsequent observations are planned for the period when Darksat is once again visible from northern Chile, including observations aimed at estimating the BRDF of Darksat and other Starlink satellites.

Acknowledgements. We would like to thank the two referees, Dr. Gregg Wade and Dr. Olivier Hainaut, for their helpful comments which improved the quality of this work. This work was supported by a CONICYT / FONDECYT Postdoctoral research grant, project number: 3180071 . JTR thanks the Centro de Astronomía (CITEVA), Universidad de Antofagasta for hosting the CONICYT / FONDECYT 2018 Postdoctoral research grant. EU kindly acknowledges the work of Marco Rocchetto and Stephen Fossey to set up Ckoirama. We are grateful to both Dr. Patrick Seitzer and Dr. Tony Tyson for useful comments on the manuscript and their encouragement. We extend a special thanks to Boris Haeussler, Felipe Gaete, Steffen Mieske, Stéphane Brilliant, Joseph Anderson, ESO Paranal, for observations which will be included in a follow-up manuscript. The following internet-based resources were used in the research for this paper: the NASA Astrophysics Data System; the ESO Online Digitized Sky Survey, the SIMBAD database and VizieR catalogue access tool operated at CDS, Strasbourg, France; and the ar $\chi$ iv scientific paper preprint service operated by Cornell University.

\section{References}

Allen, C. W. 1973, Astrophysical Quantities (London: University of London, Athlone Press)

Fukugita, M., Ichikawa, T., Gunn, J. E., et al. 1996, AJ, 111, 1748

Gallozzi, S., Paris, D., Maris, M., Scardia, M., \& Dubois, D. 2020, ArXiv e-prints [arXiv:2003.05472]

Hainaut, O. R., \& Williams, A. P. 2020, A\&A, in press, https://doi .org/10. 1051/0004-6361/202037501

Høg, E., Fabricius, C., Makarov, V. V., et al. 2000, A\&A, 355, L27

Jester, S., Schneider, D. P., Richards, G. T., et al. 2005, AJ, 130, 873

Lewis, R. R. 1994, Comput. Graphics Forum, 13, 109

McDowell, J. C. 2020, ApJ, 892, L36

Minnaert, M. 1941, ApJ, 93, 403

Munari, U., Henden, A., Frigo, A., et al. 2014, AJ, 148, 81

Phong, B. T. 1975, Commun. ACM, 18, 311

Stamnes, K., Thomas, G. E., \& Stamnes, J. J. 1999, Radiative Transfer in the Atmosphere and Ocean, 2nd edn. (Cambridge University Press)

Zacharias, N., Urban, S. E., Zacharias, M. I., et al. 2004, AJ, 127, 3043 